\title{
Star-Forming Regions in the SMC
}

\author{
I. Gonidakis ${ }^{1}$, E. Livanou ${ }^{1}$, E. Kontizas ${ }^{2}$, U. Klein ${ }^{3}$, M. Kontizas ${ }^{1}$, \\ D. Kester ${ }^{4}$, Y. Fukui ${ }^{5}$, N. Mizuno ${ }^{5}$, and P. Tsalmantza ${ }^{1}$ \\ ${ }^{1}$ Dpt. of Astrophysics, Astronomy \& Mechanics, Univ. of Athens, GR-15783 Athens, Greece \\ email: elivanou@phys.uoa.gr \\ ${ }^{2}$ National Observatory of Athens, P.O. Box 20048, GR-11810 Athens, Greece \\ ${ }^{3}$ Radioastronomisches Inst. der Universität, Bonn, Auf dem Hugel 71, 53121 Bonn, Germany \\ ${ }^{4}$ SRON, Netherlands Inst. for Space Research, P.O. Box 800, 9700 AV Groningen, Netherlands \\ ${ }^{5}$ Department of Astrophysics, Nagoya University, Chikusa-ku, Nagoya 464-8602, Japan
}

\begin{abstract}
SMC has been going through an active star formation epoch, especially during the last 0.2 Gyr when the close encounter with the LMC occured. Our goal is to detect regions dominated by early-type stars and gas and examine their behaviour at different wavelengths. Spectral energy distributions, a colour-magnitude diagram and a two-colour diagram from IRAS data (Bontekoe, Koperet \& Kester (1994); Bontekoe, Kester, Stanimirović, et al. (1999)) for these regions were used in order to compare their properties with those of starburst galaxies (Helou (1986); Lehnert \& Heckman (1995)). We have selected 50 stellar complexes with increased $100-\mu \mathrm{m}$ IRAS flux, with detetected emission in all IRAS bands and/or high concentration of young stars. Ranking them by size (Maragoudaki, Kontizas, Kontizas, et al. (1998)), a total of what we call 24 aggregates, 23 complexes and 3 super-complexes were found. Radio continuum maps at 8.6-GHz (Haynes, Murray, Klein, et al. $(1986)$ ) and the CO $(1 \rightarrow 0)$ line (Mizuno, Rubio, Mizuno, et al. (2001)) were also correlated with the map of the complexes. Only 8 of them show enhanced star formation activity according to their IR properties and $8.6-\mathrm{GHz}$ map, however, none of them resembles the IR behaviour of starburst regions found in the LMC and starburst galaxies (Livanou, Kontizas, Gonidakis, et al. (2006)). The south-west part of the "bar" has the most diverse intensity of star formation, with CO emission coincident with the largest structure. In the north-eastern end of the "bar", star formation is likely to have commenced in the recent past, with molecular gas being abundant in this region. Ongoing and future star formation are revealed in the wing, while it appears to have ceased in the central "bar".
\end{abstract}

Keywords. galaxies: structure, Magellanic Clouds, stars: formation.

\section{Acknowledgements}

The authors wish to thank the G.S.R.T. for financial support. The project is co-funded by the European Social Fund and National Resources-(EPEAEK II) PYTHAGORAS II.

\section{References}

Bontekoe, Tj.R., Koper, E. \& Kester D.J.M. 1994, A\&A 284, 1037.

Bontekoe, Tj.R., Kester, D.J.M., Stanimirović, S., Staveley-Smith, L., \& van der Hulst J.M. 1999, IAU Symposium vol. 190, p. 122.

Haynes, R.F., Murray, J.D., Klein, U., \& Wielebinski, R. 1986, A\&A 159, 22.

Helou, G. 1986, ApJ 311, L33.

Lehnert, M.D. \& Heckman, T.M. 1995, ApJS 97, 89.

Livanou, E., Kontizas, M., Gonidakis, I., Kontizas, E., Maragoudaki, F., Oliver, S. Efstathiou, A., \& Klein, U. 2006, A\&A 451, 431.

Maragoudaki, F., Kontizas, M., Kontizas, E., Dapergolas, A., \& Morgan, D.H. 1998, A\& A 338, 29.

Mizuno, N., Rubio, M., Mizuno, A., Yamaguchi, R., Onishi, T., \& Fukui, Y. 2001, PASJ 53, $45 \mathrm{~L}$. 